ACCEPTED MANUSCRIPT

\title{
From plasma to nanoparticles: optical and particle emission of a spark discharge generator
}

To cite this article before publication: Attila Kohut et al 2017 Nanotechnology in press https://doi.org/10.1088/1361-6528/aa8f84

\section{Manuscript version: Accepted Manuscript}

Accepted Manuscript is "the version of the article accepted for publication including all changes made as a result of the peer review process, and which may also include the addition to the article by IOP Publishing of a header, an article ID, a cover sheet and/or an 'Accepted

Manuscript' watermark, but excluding any other editing, typesetting or other changes made by IOP Publishing and/or its licensors"

This Accepted Manuscript is @ 2017 IOP Publishing Ltd.

During the embargo period (the 12 month period from the publication of the Version of Record of this article), the Accepted Manuscript is fully protected by copyright and cannot be reused or reposted elsewhere.

As the Version of Record of this article is going to be / has been published on a subscription basis, this Accepted Manuscript is available for reuse under a CC BY-NC-ND 3.0 licence after the 12 month embargo period.

After the embargo period, everyone is permitted to use copy and redistribute this article for non-commercial purposes only, provided that they adhere to all the terms of the licence https://creativecommons.org/licences/by-nc-nd/3.0

Although reasonable endeavours have been taken to obtain all necessary permissions from third parties to include their copyrighted content within this article, their full citation and copyright line may not be present in this Accepted Manuscript version. Before using any content from this article, please refer to the Version of Record on IOPscience once published for full citation and copyright details, as permissions will likely be required. All third party content is fully copyright protected, unless specifically stated otherwise in the figure caption in the Version of Record.

View the article online for updates and enhancements. 
Abstract. The increased demand for high purity nanoparticles of defined geometry necessitates the continuous development of generation routes. One of the most promising physical techniques for producing metal, semiconductor or alloy nanoparticles in the gas phase is spark discharge nanoparticle generation. The technique has a great potential for up-scaling without altering the particles. Despite the simplicity of the setup, the formation of nanoparticles in a spark discharge takes place via complex multi-scale processes, which greatly hinders the investigation via conventional nanoparticle measurement techniques. In the present work, time-resolved optical emission spectroscopy was used to provide information on the species present in the spark from as early as approximately $100 \mathrm{~ns}$ after the initiation of the discharge. We demonstrate that operando emission spectroscopy can deliver valuable insights into nanoparticle formation. The emission spectra of the spark are used to identify, among others, the main stages of material erosion and to calculate the quenching rate of the generated metal vapour. We demonstrate that the alteration of key control parameters, that are typically used to optimize nanoparticle generation, clearly affect the emission spectra. We report for $\mathrm{Cu}$ and $\mathrm{Au}$ nanoparticles that the intensity of spectral lines emitted by metal atoms levels off when spark energy is increased above an energy threshold, suggesting that the maximum concentration of metal vapour produced in the generator is limited. This explains the size variation of the generated nanoparticles. We report a strong correlation between the optical and particle emission of the spark discharge generator, which demonstrate the suitability of optical emission spectroscopy as a valuable characterization tool that will allow for the more deliberate optimization of spark-based NP generation.

Keywords: spark discharge, nanoparticle generation, optical emission spectroscopy, plasma synthesis 


\section{Introduction}

The mass-production of nanoparticles (NPs) by environmentally friendly and cheap means is a crucial technological bottle-neck if nanoparticle-based product development should continue to advance. Physical methods offer a suitable alternative to chemical means to fulfil these requirements, by exhibiting inherent advantages over competing methods, such as continuous generation, high purity of the NPs, smaller amount of waste and more straightforward scalability (Biskos et al 2008).

Flame pyrolysis is an established method used for industrial scale production of metal-oxide particles (Wegner and Pratsinis 2003), but unsuitable for the production of pure metal particles. Metal NPs are often formed by material evaporation in an inert gas followed by subsequent nucleation and condensation (Meuller et al 2012). Evaporation can be achieved in several ways, e.g. using furnace (Magnusson et al 1999), glowing wires (Schmidt-Ott et al 1980), or laser ablation (Kato 1976). These methods are, however, not energy efficient and their upscaling for mass-production is problematic. The most energy efficient and demonstrably up-scalable method for the evaporation of conducting materials is spark discharge generation (Schwyn et al 1988, BUONAPART-E).

The spark discharge generator (SDG) has an appealingly simple design that consists of a leak-tight chamber, housing two electrodes that are separated by a small gap. For creating a high voltage spark discharge between the electrodes, a self-pulsed circuit is typically used, consisting of a capacitor fed by a high voltage DC power supply (charging loop), that is connected in parallel to the electrode gap (discharge loop). Each spark is initiated when the gaseous ambient in between the electrodes breaks down, i.e. via the formation of a conducting channel (spark channel) between the two electrodes in which the charge carriers are initially dominated by electrons and ions of the carrier gas. The colloquial term "single spark" suggests a simple physical process but actually refers to an oscillatory event, in which the amplitude of the sinusoidal voltage and current signals is damped exponentially and hence the polarity also varies periodically (Hontanón et al 2013). The surfaces of the electrodes are heated at the so called "hot spots" where the spark channel interacts with the electrode surfaces and hence the eroded material of the electrodes forms a vapour plume (Pfeiffer et al 2014, Borra 2006). In addition to this, ions of the carrier gas, as well as those of the anode and the cathode bombard the electrodes that may lead to further removal of electrode material (Borra et al 1998). When the vapour plume is cooled by adiabatic expansion and mix with the carrier gas nucleation will take place, which forms atomic clusters, at first. These clusters will grow further into singlet particles by condensation and coalescence and ultimately grow to primary particles that may, eventually, form agglomerate particles if dilution is not sufficiently rapid (Pfeiffer et al 2014).

The potential of spark discharge for mass-production of NPs is only partly based on its relative simplicity. More importantly, the generation process could be easily and controllably scaled up by placing several electrode pairs in parallel at low cost and with minimal impact on the environment. In the BUONAPARTE project, 21 partners from bothindustry and academia worked on this upscaling approach and realized a production rate of $100 \mathrm{~kg}^{-1}{ }^{-1}$ (BUONAPART-E).

Although the SDG has been around us since the invention of the gasoline engine, its first use as an intentional nanoparticle generator dates to 1988 (Schwyn et al 1988), which is now commercially available (Helsper et al 1993), and used by several groups (Messing et al 2010a, Messing et al 2010b, Kala et al 2016, Pfeiffer et al 2015, Bae et al 2017, Muntean et al 2016, Wagner et al 2016, Byeon et al 2009), the very fundamentals of the processes leading to particle formation is not yet fully understood. One reason for this knowledge gap lies in the difficulty of investigating the different stages of nanoparticle formation, especially the initial, early phase of the process. Traditionally, there are two main 


\section{From plasma to nanoparticles: optical and particle emission of a spark discharge generator \\ AUTHOR SUBMITTED MANUSCRIPT - NANO-115013.R1}

approaches in aerosol science, through which gas-borne particles are characterized. One of this is NP collection (e.g. sampling on various substrates by using electrostatic precipitators, impactors, etc.) followed by off-line electron microscopic analysis. In the other approach, aerosol instrumentation capable of in-flight measurements is used, such as the scanning mobility particle sizer (SMPS), aerosol particle mass analyzer (APM), aerosol mass spectrometer (AMS) or laser-vaporization aerosol mass spectrometer (LV-AMS) (Nilsson et al 2015). However, all of these apparatuses are only applicable further downstream of the generator spark gap, and none can acquire fundamental information in the vicinity of the primary NP formation zone. Invasive methods, such as proximity particle sampling (i.é. close to the spark gap) have been attempted for bridging this knowledge gap (Ludvigsson et al 2015). However, proximity sampling always $i$ ) provides integrated information on the early stages of particle formation and ii) the flow field and hence the generation process itself is altered by the sampling.

In this paper, we employ a non-invasive method, namely time resolved optical emission spectroscopy (OES) for gaining information on the processes preceding particle formation in the spark without disturbing the examined processes by any means. The outstanding potential of OES for the investigation of electric discharges is well reflected by the fact that the technique has a sound literature in connection with spectroanalytical chemistry (Boumans 1972, Scheeline 1990). This technique allows for the in situ and operando investigation of the spark plasma with a temporal resolution on the nanosecond time-scale. OES provides direct information on the atomic and ionic species which populate the spark plasma right before the NP nucleation process. Time-resolved emission spectra can be used to derive key plasma properties, such as the temperature and electron concentration (Kohut et al 2017), which are crucial for the parametrization and validation of various particle growth models. In the present work, time resolved OES was also used to identify the main stages of the material erosion and to calculate the quenching rate of the generated metal vapour. We also demonstrate that the alteration of key control parameters, that are typically used to optimize nanoparticle generation, e.g. gap size and charging current, clearly affect the emission spectra. Consequently, the atomic and ionic species are, though specific to the very early stages of the process, i.e. precede nucleation, significantly influence the NP generation route. More specifically, in the present study, the effect of charging current and the size of the electrode gap on the erosion of copper and gold electrodes was investigated by means of OES, while generated NPs were characterized downstream by an SMPS. Correlations between particle characteristics and the optical emission are also presented. Our findings contribute significantly to the understanding of the early stages of particle formation in an SDG and will lead to a more deliberate optimization of spark-based NP generation.

\section{Methods}

\subsection{Experimental setup}

The SDG used in this work consists of a Technix CCR15-P-150 capacitance charger high voltage power supply, that charges a $20 \mathrm{nF}$ high voltage capacitor bank. The voltage is carried to the initially anodic electrode via a high voltage cable. The opposite electrode is connected to the ground via a separate cable. Please note that from this point forward, the two electrodes will be identified according to their initial electrical polarity that is anode and cathode. The spark chamber consists of a standard KF40 6-way cross with the gas inlet and outlet on the top and bottom flanges, respectively (the experimental setup is schematically depicted in the Supplementary data in figure S6). Two horizontal, opposing flanges are used for the electrical feedthroughs, connected internally to the cylindrical electrodes aligned uniaxially. A high yoltage feedthrough (for the anode) and a linear motion manipulator (for the cathode), both manufactured by MDC Vacuum Products, was used in the experiments. Gas control was realized by using 
an AERA FC-7700CU mass flow controller. Pressure control was achieved with a Bronkhorst P-702CV electronic controller. The SDG system is controlled via purpose-made LabView software through a National Instruments cDAQ with a NI-9205 module for analog input, a NI-9264 module for analog output and a NI-9403 module for digital input and output. The discharge voltage is continuously monitored by using a Tektronix P6015A high-voltage probe that has a bandwidth of $75 \mathrm{MHz}$ and capability of measuring voltage peaks up to $40 \mathrm{kV}$.

The SDG is connected to a scanning mobility particle sizer (SMPS) setup constructed from a particle charger containing a $\beta$-emitting ${ }^{63} \mathrm{Ni}$ source and a dynamic mobility analyzer (TSI 3081). An electrostatic precipitator was used to facilitate controlled deposition of NP samples for ex-situ characterization by means of transmission electron microscopy (TEM).

Copper (99.9\% purity) and gold (99.9\% purity) electrodes with flat surfaces and diameters of $3 \mathrm{~mm}$ were eroded via sparking. All measurements were performed under ambient pressure with nitrogen $(99.995 \%$ purity) as carrier gas at a flow rate of $1.68 \mathrm{slm}$. The electrode distance and the charging current were varied in the range of 1.0-4.0 $\mathrm{mm}$ and 2-15 $\mathrm{mA}$, respectively.

The time-resolved OES measurements were carried out by an Andor Mechelle 5000 fibre-coupled echelle spectrograph equipped with an Andor iStar 734-18F-03 intensified CCD (ICCD) camera. In order to reduce the electromagnetic interference induced by the SDG, the spectroscopic instrumentation was set up in a nearby laboratory and the optical signal was transmitted to the spectrometer using a $12 \mathrm{~m}$ long fused silica optical fibre with $50 \mu \mathrm{m}$ core diameter. The emitted light from the plasma was spatially integrated over a ca. $6 \mathrm{~mm}$ diameter circular area (in a direction perpendicular to the axis of the electrodes) by using a quartz collection lens (Avantes COL-UV/VIS). The position of the collecting lens was optimized (to the highest optical signal) before each experimental run. The spectrograph was wavelength and intensitycalibrated and allowed for spectral data collection in the $300-800 \mathrm{~nm}$ wavelength range with $\sim 0.125 \mathrm{~nm}$ resolution (at $435 \mathrm{~nm}$ ). The light acquisition of the ICCD camera was triggered by the sharp negative signal edge (provided by the HV probe) caused by the breakdown of the gas between the electrodes. A Stanford DG535 pulse generator was used to produce a logical signal after the onset of the breakdown and initiated the exposure, i.e. the spectral acquisition. The overall time delay (including insertion delays and signal propagation) of the spectroscopic setup caused is around $100 \mathrm{~ns}$, which thus defines the temporal starting point of our investigations. In order to study the time evolution of the spark, emission spectra of the discharge were acquired at time delays varying from 0 ns to $15 \mu \mathrm{s}$. The gate width of the camera, hence the temporal resolution of the measurements, was set to $50 \mathrm{~ns}$. Each spectrum was measured on separate, consecutive sparks and few hundred spectra were accumulated at each experimental setting.

It should be noted that by the OES technique one can only gain information on those species that emit light detectable to the spectrometer used. A practical consequence of this is that the temporal domain accessible by OES is jointly set by the peculiarities of the SDG (especially those that affect when the species got ionized and/or excited in the gap) and the spectroscopic properties of the particular chemical system under investigation (e.g. excitation and relaxation times of species). When one needs to extend the temporal domain beyond this limit (which is about $20 \mu \mathrm{s}$ in our case) further spectroscopic techniques might be implemented to facilitate the investigation of non-emitting species (e.g. relaxed atoms). An obvious choice would be optical absorption spectroscopy (OAS), when the absorption of the light intensity of a finely tuned excitation source is detected (Galbács 2006). OAS is an excellent selective detection scheme for widening the temporal window of optical investigation, but would inevitably 


\section{Results}

\subsection{Optical emission of the spark}

Erosion of the electrode material in an SDG is driven by the electrical discharge formed in the electrode gap. Due to the high energy input, not only atoms are removed from the electrodes but these atoms will also get excited and, if temperature permits, ionized. The characteristic optical emission originates from the relaxation of these excited atoms and ions. Figure 1 represents a typical spark emission spectrum acquired by spatially integrating the photons leaving the electrode gap into the acceptance angle of the lens. The temporally resolved spectra were summed in order to illustrate the spectral character of the overall spark emission.

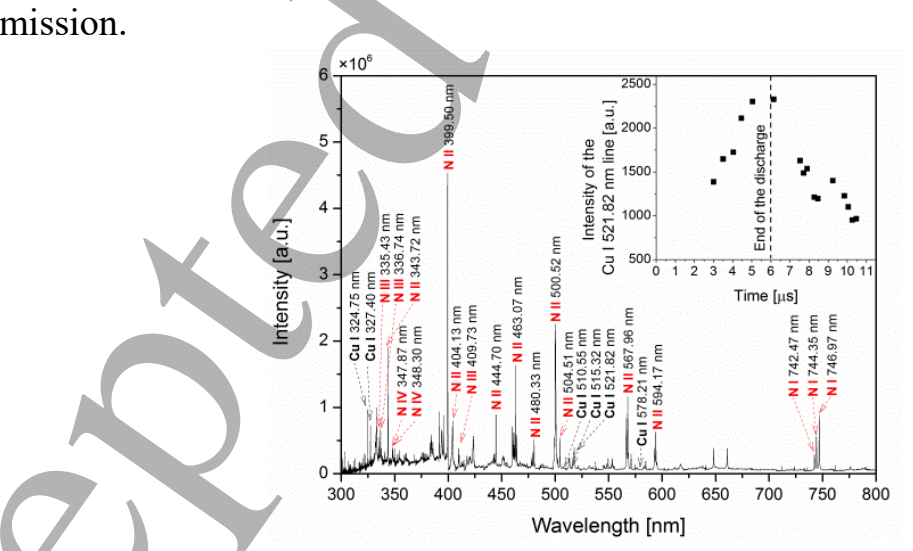

Figure 1 Temporally and spatially integrated emission spectrum of the spark acquired during the generation of $\mathrm{Cu}$ NPs in nitrogen atmosphere (4 mm gap, $1.68 \mathrm{slm}$ gas flow rate, $10 \mathrm{~mA}$ charging current). Inset: temporal evolution of the intensity of the $\mathrm{Cu}$ I $521.82 \mathrm{~nm}$ line (2 mm gap, $1.68 \mathrm{slm}$ gas flow rate, $15 \mathrm{~mA}$ charging current).

As can be seen in figure 1, the spectrum is dominated by the emission of the excited species of the gas ambient, namely spectral lines of nitrogen atoms and singly or multiply charged $\mathrm{N}$ ions. Atomic lines of the electrode material $(\mathrm{Cu})$ are also present with much smaller relative intensity, which is a common 
feature of spectra acquired in the SDG. This relation reflects that the number concentration of the gas species exceeds the number concentration of the electrode material in the spark gap by several orders of magnitude (Kohut et al 2017).

As a single spark has a characteristic temporal behaviour (the oscillatory spark discharge has an alternating voltage and current waveform with exponentially damping amplitude, see e.g. in (Palomares et al 2016)) the quantity and the properties (e.g. temperature) of the eroded material evolves in time as well. This temporal evolution is well reflected in the emitted spectrum.

The optical emission of spark discharges used for spectroanalytical purposes has a well-established literature dating back to the last century (Boumans 1972, Walters 1969, Scheeline and Coleman 1987). The evolution of an electric spark discharge is divided into four stages: $i$ ) pre-breakdown, ii) breakdown, iii) arc and iv) afterglow (Boumans 1972). The pre-breakdown and breakdown stages are responsible for the increase of charge carriers in the electrode gap, which ultimately form a conducting plasma channel that bridges the two electrodes (Raizer 1991). In the arc stage, the spark very much behaves like a transient arc discharge (Boumans 1972, Mandelstam 1959). After the electric current ceased, the spark enters the so-called afterglow regime. The name of this final stage reflects the observation that the species emit light for an extended period of time, i.e. well after the conductivity of the electrode gap diminishes. The pre-breakdown and breakdown stages have negligible contribution to the amount of eroded material which is supported by the fact that the emission spectrum of these stages are characterized by molecular bands when a molecular gas (e.g. $\mathrm{N}_{2}$ ) is used as ambient gas (Walters and Malmstadt 1965). In the typical, i.e. free-running SDG, the breakdown is expected to complete quickly due to the low inductance of the discharge loop (Walters and Malmstadt 1965). Figure 1 also suggests that the breakdown completes in less than $100 \mathrm{~ns}$, since the spectral acquisition started about $100 \mathrm{~ns}$ after the voltage drop between the electrodes, and do not exhibit molecular nitrogen bands. This behaviour is in line with the $\sim 50 \mathrm{~ns}$ breakdown duration value reported recently in case of an SDG operated under argon atmosphere (Kohut et al 2017). This means that the duration of the breakdown is negligible as compared to the timescale of the entire emission process. After the breakdown, alternating current starts to flow in the gap with exponentially damped amplitude, and the spark enters the arc stage. By the end of the arc stage, the capacitor fully discharges. The majority of electrode erosion takes place in this stage, when electric energy is pumped into the electrode gap. The numerous ionic nitrogen lines shown in figure 1 correspond to this stage (Boumans 1972), which is the direct evidence of the high temperature and electron concentration characteristic to the electrode gap. The duration of the arc stage is determined by the resistance, capacitance and inductance of the discharge loop and is about $6 \mu$ s for the present SDG. Even though the generation of metal vapour is less significant after this stage, the previously atomized material still has a rather high temperature as evidenced by the prolonged light emission from the spark gap. The temporal evolution of the intensity of a typical atomic copper line $(521.82 \mathrm{~nm})$ is also shown in the inset of figure 1. It can be seen that the emission intensity peaks around $6 \mu$ s that is about the end of the arc stage and also when material erosion terminates. After this point in time, the intensity of the emission monotonously decreases and finally fades away in about 11-12 $\mu$ s after the onset of breakdown. The emission spectrum acquired in the afterglow phase is not influenced by erosion, only by the conditions of the discharge-free gap. Further details on the temporal evolution of the emission spectrum of the spark plasma are given in the Supplementary data.

In the following, the effect of gap size (the distance between the electrodes) and charging current (used for replenishing the discharged capacitor) will be shown on the properties of various species of the electrode material and the carrier gas, present in the spark gap. In order to prove the generality of our 
approach, all experiments were performed using two electrode materials, namely $\mathrm{Au}$ and $\mathrm{Cu}$, exhibiting rather different erosion properties (Tabrizi et al 2009). Results for $\mathrm{Cu}$ electrodes will be shown in the main manuscript, while detailed results obtained for Au electrodes are given in the Supplementary data. Although monitoring the emission of metal atoms is most directly related to NP generation, along with that the emission of atomic nitrogen, the most abundant component of the spark plasma is also discussed here. To this end, the intensity (which is defined as the area under the spectral line) of selected atomic copper, gold and nitrogen lines $(521.82 \mathrm{~nm}, 479.26 \mathrm{~nm}$ and $746.83 \mathrm{~nm}$, respectively) was measured in the afterglow stage of the spark (more precisely, in the temporal window of about 5-15 $\mu$ s after the onset of the breakdown). The temporally resolved spectra were summed in order to get a cumulated spectrum representing the afterglow stage.

The intensity of spectral lines for the atomic species of the electrode and the background gas follows very similar trends in case of both $\mathrm{Cu}$ and $\mathrm{Au}$ electrodes. Figure 2A shows the variation of the normalized intensity (see the Supplementary data for details) of an atomic $\mathrm{Cu}$ and $\mathrm{N}$ line as a function of charging current. Atomic nitrogen emission decreases monotonously, while the emission of copper atoms varies via a wide maximum when charging current is increased.
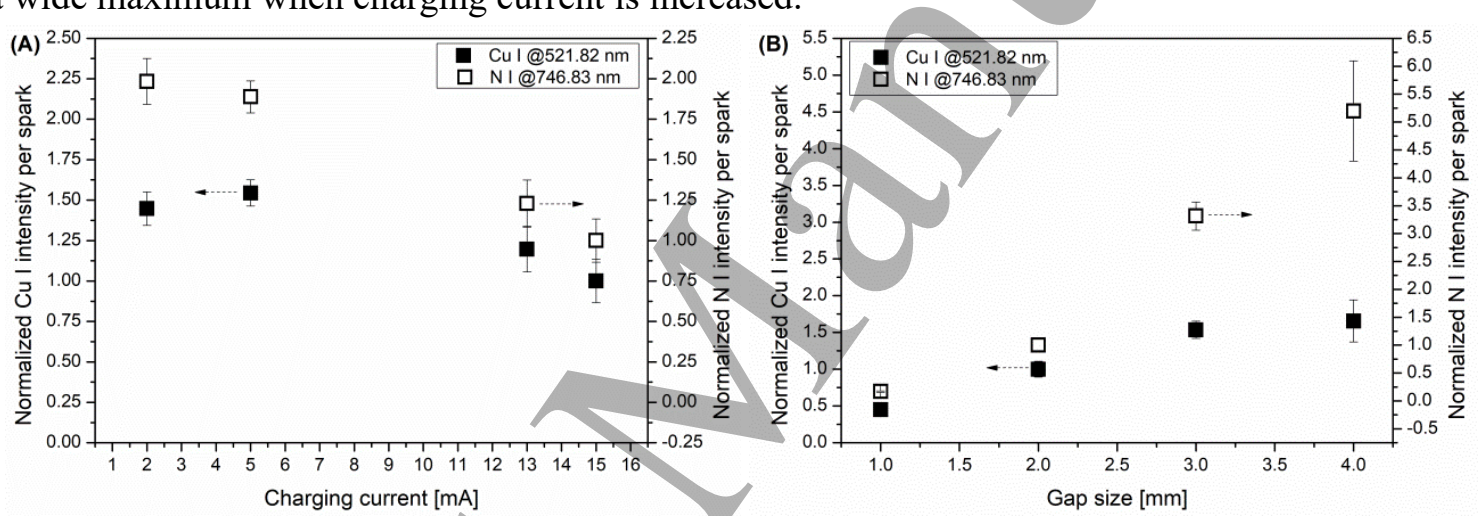

Figure 2 Normalized intensity of an atomic nitrogen and copper line as a function of the charging current (A) and gap size (B) acquired during sparking between $\mathrm{Cu}$ electrodes.

(2 mm gap size, $1.68 \mathrm{slm}$ gas flow rate).

The variation of gap size results in a different trend. As it is shown in figure $2 \mathrm{~B}$, the intensity of the N I (for the definition of spectroscopic notations see the Supplementary data) line increases monotonously with increasing gap size, while the emission of copper atoms shows saturation at about $3 \mathrm{~mm}$ gap sizes. Figure 2 proves that the key control parameters of the SDG (such as the gap size and the charging current) clearly affect the optical emission of the spark. Similar results were obtained in nitrogen atmosphere using Au electrodes (cf. figure 2 and figure S2 in Supplementary data).

\subsection{Nanoparticles emitted by the $S D G$}

The nanoparticle production of the SDG was characterized by an SMPS. Figure 3A illustrates the effect of charging current on the size distribution of $\mathrm{Cu}$ NPs. The mobility size ${ }^{*}$ distribution of the NPs follows log-normal distribution with a modus in the range of $18-30 \mathrm{~nm}$. Both the modal diameter and peak

${ }^{*}$ In aerosol science, particles are often sized by their electrical mobility diameter, which is an equivalent diameter assigned to the particles according to their mobility in the electric field, e.g. experienced in an SMPS 


\section{Discussion}

\subsection{Quenching rate and particle formation}

The intensity of the atomic copper line at $521.82 \mathrm{~nm}$ measured as a function of time is replotted in figure 4. The temporal evolution of the spectral line intensity suggests that either the concentration or the temperature of the excited species (or both) is decreasing after $6 \mu \mathrm{s}$. Using the Boltzmann plot method, the plasma temperature can be calculated from the temporally resolved emission spectroscopic data (see Methods section). As it is shown in figure 4 (solid symbols), the instantaneous temperature describing the plasma when the electrode material is most abundant in the gap was found to be about $12000 \mathrm{~K}$, which decreases linearly down to ca. $9000 \mathrm{~K}$ in the temporal window in which the emission of atomic copper was detectable with a reasonable signal-to-noise ratio.

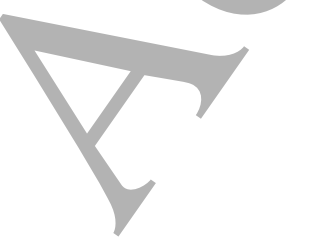




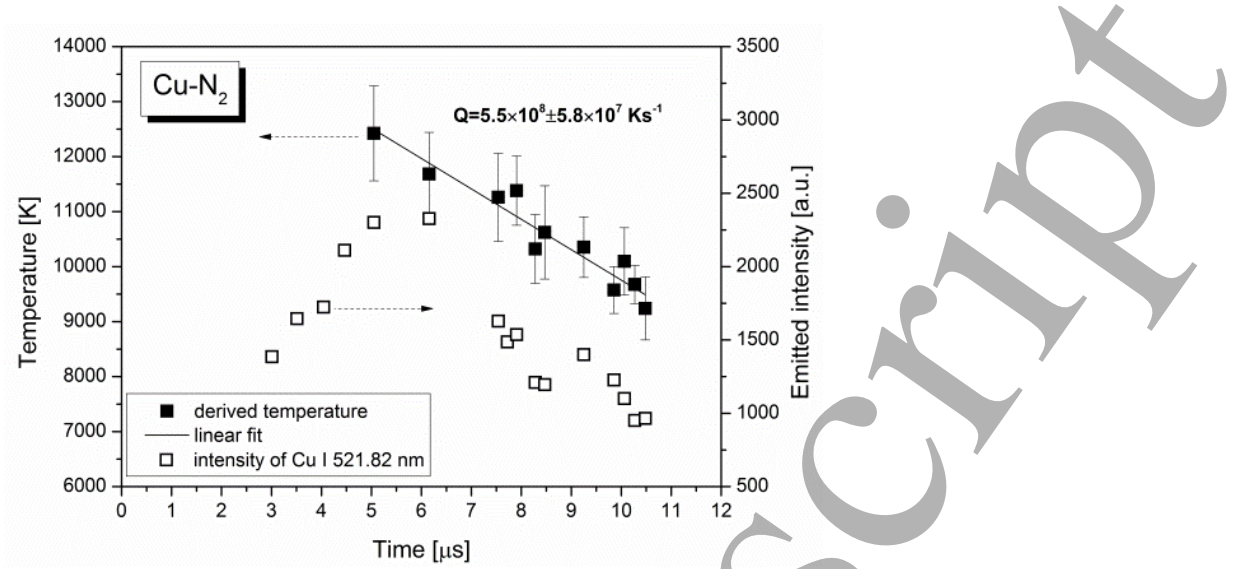

Figure 4 The temporal evolution of the intensity of the $\mathrm{Cu}$ I $521.82 \mathrm{~nm}$ line (open squares) and the evolution of the temperature of the spark plasma derived from the emission spectra (full squares) ( $2 \mathrm{~mm}$ gap, $1.68 \mathrm{slm}$ gas flow rate, $15 \mathrm{~mA}$ charging current).

The rate of temperature decrease, the so-called quenching rate, was estimated to be $(5.5 \pm 0.6) \times 10^{8} \mathrm{Ks}^{-1}$. This value fits into the $10^{6}-10^{9} \mathrm{Ks}^{-1}$ range reported in the literature (Feng et al 2016, Berkowitz and Walter 1987, Jenkins and Eagar 2003, Martinen and Toll 1970), although it is closer to the higher end of the literature range. Recently Feng et al. estimated the quenching rate in an SDG for gold vapour in nitrogen atmosphere and obtained $7.5 \times 10^{6} \mathrm{Ks}^{-1}$ (Feng et al 2016), which is almost two orders of magnitude smaller than the value we report here. They assume that the temperature of the atomic gold vapour is around the boiling point, however as we found it here by evaluating the optical emission spectrum of the spark, the temperature is considerably higher than that, even at later stages of the spark discharge. Thus, we believe that values of about $10^{8}-10^{9} \mathrm{Ks}^{-1}$, including our own, are more accurate quenching rate estimates.

Knowing the quenching rate and the temperature at the end of the arc stage, one can estimate how long it takes the metal vapour to cool down to room temperature, which is about $25 \mu$ s for copper NPs in nitrogen atmosphere at a gap size of $2 \mathrm{~mm}, 1.68 \mathrm{slm}$ gas flow rate and charging current of $15 \mathrm{~mA}$. This can be considered as an estimate for the end of the cooling stage, and also the starting point of particle formation. By considering the typical $50-200 \mathrm{~Hz}$ spark repetition rate values employed in the present study, which result in spark events in the millisecond characteristic range and the above cooling time which is a few tens of microseconds, one can conclude that NPs form independently due to consecutive spark. By adopting the main mechanisms relevant to particle formation (nucleation, coagulation, turbulent dilution, turbulent diffusion, laminar diffusion, aggregation) and their respective approximate time domains from (Feng et al 2016) including those of nanoparticle formation can be summarized as it is shown in figure 5. The schematic spectra shown at the top of figure 5 represents the emission characteristics typical to the material erosion and the cooling phases. Due to the high (in the order of 100 $\mathrm{mJ}$ ) energy pumped into the gap during the arc stage of the spark the spectrum is dominated by gas ions while the emission acquired in the cooling stage is characterized by atomic copper and nitrogen emission. As a result of particle growth steps (schematically shown at the bottom of figure 5) aggregated $\mathrm{Cu}$ nanoparticles (shown in the right inset of figure 5) form from the $\mathrm{Cu}$ atoms eroded from the electrodes in the material erosion phase. 


\subsection{Effect of control parameters}

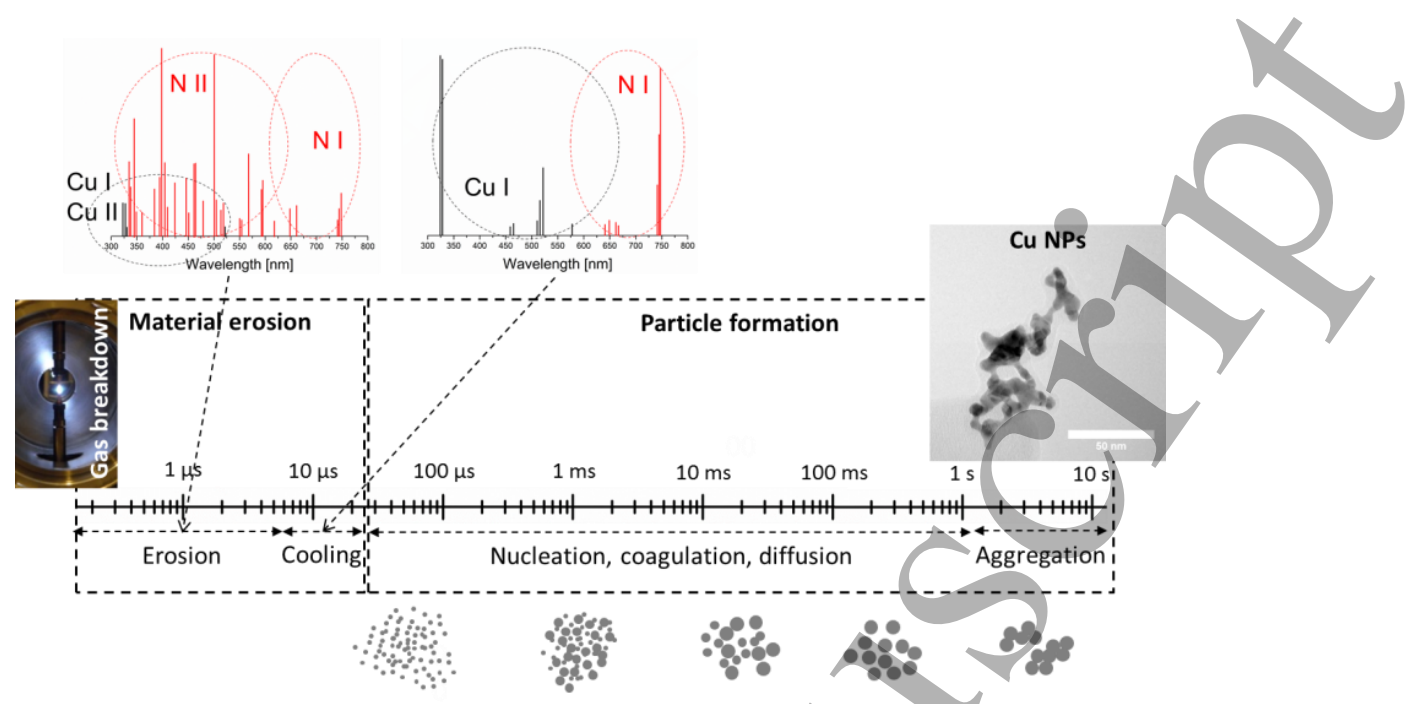

Figure 5 Approximate time scale of NP formation in SDGs from the breakdown of the gap to the formation of NP aggregates. The typical spectral character of the material erosion is shown together with the main NP formation mechanisms and time-range as adopted from34. A typical $\mathrm{Cu}$ NP aggregate is shown in the right inset.

In a conventional SDG setup, also used here, the main control parameters are the flow rate of the carrier gas, the gap size and the charging current. These parameters affect the NP output of the generator the most via tuning the condensation process by controlling the energy and the repetition rate of the sparks (Feng et al 2015).

The energy per spark is proportional to the energy/stored in the capacitor right before the breakdown (Tabrizi et al 2009). Hence the energy accumulated in the capacitor is:

$$
E=\frac{1}{2} C U_{b d}^{2}
$$

where $C$ is the capacitance and $U_{b d}$ is the breakdown voltage. At a constant pressure and electrode geometry the breakdown voltage predominantly depends on the gap size according to Paschen's law (Fridman and Kennedy 2011), hence the energy per spark is proportional to the square of the gap size.

At a fixed gap, the spark repetition rate (SRR) can be varied by changing the charging current as described by (Tabrizi et al 2009):

$$
S R R=\frac{I_{c h}}{C U_{b d}}
$$

where $I_{c h}$ is the charging current of the capacitor, supplied by the power supply. It should be noted that the increase of SRR results in decreasing breakdown voltage and hence decreasing spark energy. This is most probably due to the fact that charge carriers are left behind by the preceding spark and accumulated in the gap thereby somewhat lowering the breakdown voltage as compared to the single spark or low frequency cases (Crawford and Edels 1960).

The variation of breakdown voltage as a function of gap size and charging current is plotted in figure 6A for sparking between $\mathrm{Cu}$ electrodes. As can be seen in figure 6A the breakdown voltage is increasing linearly with increasing gap size in qualitative agreement with Paschen's law (Fridman and Kennedy 2011). The increasing charging current and hence increasing SRR results in a linearly decreasing breakdown voltage. The energy per spark calculated from the breakdown voltage using (1) and the 
measured SRR is shown in figure 6B and C, respectively both as a function of gap size and charging current. On one hand, the energy per spark is directly determined by the variation of the breakdown voltage (figure 6A) according to (1), which therefore increases with the increase of the gap size in a quadratic manner and decreases with the increase of the charging current (figure 6B). On the other hand, the SRR decreases with increasing gap and increases quasi linearly with increasing charging current, as dictated by Eq. 2. Similar behaviour was observed for gold electrodes, which are documented in the Supplementary data (figure S4).
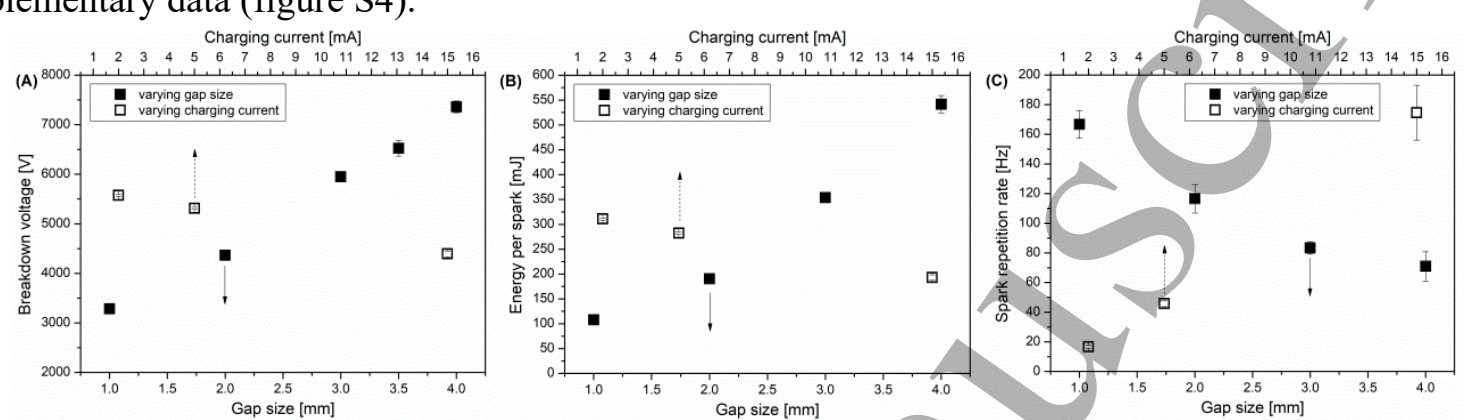

Figure 6 The variation of the breakdown voltage (A), energy per spark (B), and spark repetition rate (C) as a function of gap size at $10 \mathrm{~mA}$ charging current and as a function of the charging current at $2 \mathrm{~mm}$ gap in case of $\mathrm{Cu}$ electrodes in nitrogen atmosphere.

The tendencies shown in figure 6 are indispensable for interpreting the variation of the size distribution of the generated NPs as a function of charging current (cf. figure 3A). Qualitatively speaking, the variation of charging current causes the variation of SRR (figure 6C). Increased SRR produces more eroded material per second, which results in higher particle concentration, more pronounced aggregation and larger modal diameter of the generated NPs (Tabrizi et al 2009, Boddu et al 2011). This explains the trends shown in figure 3A, namely that both the diameter and the concentration of the generated NPs increases with increasing charging current. The SRR depends more strongly on the charging current than the spark energy does (cf. figure $6 \mathrm{~B}$ and $\mathrm{C}$ ), which results in an overall increasing trend in particle diameter.

By changing the gap size at a fixed charging current, both the SRR and the energy per spark is changing significantly. As it was shown in figure $3 \mathrm{~B}$, the diameter and the concentration of the generated NPs reach a peak value and start to decrease when the gap size is increased. This behaviour is the combined effect of the variation of SRR and energy per spark at different gaps as shown in figure 6B and C. The spark energy strongly increases with increasing gap, while the SRR decreases. The higher the energy per spark the more material is eroded from the electrodes (Tabrizi et al 2009, Horvath and Gangl 2003), which in turn increases the vapour concentration in the gap. This effect is reflected in the size distributions measured at $1 \mathrm{~mm}$ and $2 \mathrm{~mm}$ gaps for $\mathrm{Cu}$ (cf. figure 3B), and in the gap range of 1-3 mm for $\mathrm{Au}$ (cf. figure S3B). However, at larger gaps the higher energy per spark value apparently cannot compensate for the diminishing effect of the decreasing SRR (cf. figure 6C), which causes an overall decrease in the total particle concentration. This finding - which is rather unexpected in light of the strongly increasing spark energy (cf. figure 6B) - will be discussed in the following sections.

On one hand, we suggest that the size and concentration of the NP agglomerates (generated at different conditions) reflect the combined effect of SRR and energy per spark. On the other, the amount of metal vapour eroded by a single spark is defined by the energy per spark (Horvath and Gangl 2003) and OES is a perfect tool to gain in situ information on the erosion process in this regard. If we assume that the 
optical emission of the spark plasma depends solely on the energy per spark, the atomic emission intensities (shown in figure 2) can be presented on the same graph regardless of whether the particular energy per spark value was achieved via varying the gap size, the charging current, or both. Please note, that the measured absolute emission intensity values depend on the light collection conditions (e.g. the optical system, integration time, gain factor, averaging, etc.) so the spectra measured in different experimental runs were scaled to make trends comparable. Therefore each measurement series was performed to contain overlapping energy values which were used to normalize the acquired spectra to the same spark energy.

Since the carrier gas, here nitrogen, is the most abundant component in the spark gap (Kohut et al 2017), the effect of energy per spark is examined on the emission of nitrogen atoms at first. The normalized intensity of a typical spectral line of atomic $\mathrm{N}$ measured at different spark energies during sparking between $\mathrm{Cu}$ electrodes is shown in figure 7A. Points represented by closed and open symbols were obtained when the particular energy per spark value was achieved via changing the gap size, and charging current, respectively. As illustrated by the fitted dashed line, the emission intensity of atomic $\mathrm{N}$ increases in a linear fashion in the energy range studied, regardless of whether the energy per spark increase is realized via adjusting the gap or the charging current. This also justifies, though only indirectly, our previous assumption on the dependence of emission intensity on spark energy. As can be seen for $\mathrm{Cu}$ in figure 7A and in figure S5A for $\mathrm{Au}$ the observed linear correlation applies to both electrode materials, further strengthening the fundamental role of spark energy.
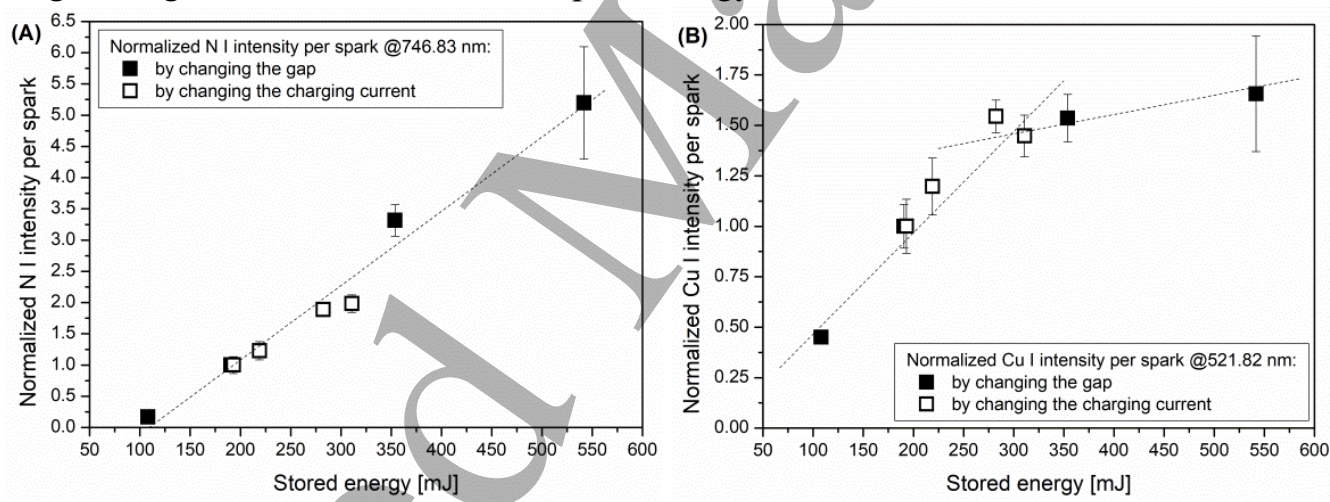

Figure 7 Normalized intensity of an atomic nitrogen (A) and copper (B) line as a function of the energy per spark acquired during sparking between $\mathrm{Cu}$ electrodes. (Varying gap and charging current values, $1.68 \mathrm{slm}$ gas flow rate.)

As shown in figure 7B, the emission of metal atoms behaves differently from that of the nitrogen. The emission intensity of $\mathrm{Cu}$ I increases roughly linearly up to about $300 \mathrm{~mJ}$ (by varying either the gap or the charging current), beyond which the increasing tendency continues to grow less steeply and eventually saturates. A very similar trend is shown for Au in figure S5B of the Supplementary data.

The intensity of a spectral line depends on the temperature and the concentration of the given species (see the Temperature calculation section in the Supplementary data for more details). Assuming that the spark plasma is in local thermodynamic equilibrium (LTE) (we recently proved that LTE holds in the afterglow stage of a spark used for $\mathrm{Cu}$ NP generation in Ar atmosphere (Kohut et al 2017)) it follows that nitrogen and metal atoms, along with every other species in the same spatial region, have the same temperature. This suggests that the difference between figure $7 \mathrm{~A}$ and $\mathrm{B}$ should be attributed to the concentration difference of nitrogen and copper. Nitrogen atoms are present in the spark gap before each spark event at 


\section{From plasma to nanoparticles: optical and particle emission of a spark discharge generator}

a constant concentration while copper or gold atoms are only produced during sparking. Therefore the dissimilar behaviour of nitrogen and copper, as evidenced by figure 7A and B implies that beyond a threshold value, energy is utilized less efficiently for increasing the concentration of metal atoms. As it is indicated in figure 6B, the ca. $300 \mathrm{~mJ}$ threshold energy (cf. figure 7B) is reached when the gap is increased from $2 \mathrm{~mm}$ to $3 \mathrm{~mm}$. This correlates well with the total concentration (and the diameter) of the generated particles - as measured by the SMPS - which started to decrease between $2 \mathrm{~mm}$ and $3 \mathrm{~mm}$ gap sizes despite of the monotonously increasing energy per spark (cf. figure 3B). An analogous behaviour was observed for Au (cf. figure S5B), with the sole exception that the threshold energy of/about $600 \mathrm{~mJ}$ corresponds to a gap of $3 \mathrm{~mm}$, correlating neatly with the peak concentration and the modal diameter of the generated Au NPs (cf. figure S3B). Although the amount of metal vapour eroded in unit time cannot be directly translated to the total particle concentration or diameter, they are related to each other. As it was shown e.g. by Byeon et al., the more material is eroded in unit time the higher the concentration and the larger the diameter of NPs will be (Byeon et al 2008).

Our OES results suggest that the concentration of metal vapour produced in an SDG only can be increased, by increasing the energy per spark, up to a certain threshold energy, either increasing the spark gap or the charging current. Therefore, it is qualitatively understandable why the total NP concentration and hence the modal diameter follow the SRR instead of the more steeply increasing energy per spark curve after a certain gap size. The generality of this finding is further strengthened by the fact that the very same behaviour was found for $\mathrm{Cu}$ and $\mathrm{Au}$ electrodes. It should be noted that the exact value of the threshold energy depends on the electrode material used. This energy is about $300 \mathrm{~mJ}$ for $\mathrm{Cu}$ and $\sim 600 \mathrm{~mJ}$ for $\mathrm{Au}$ (cf. figure 7B and figure S5B). Tabrizi et al. measured the mass loss of Au electrodes as a function of the spark energy and reported a linear trend (Tabrizi et al 2009). However, the highest energy value was about $150 \mathrm{~mJ}$ in their experiments, which is well below the energy limit derived in the present work, i.e. they worked well within the linear domain. Horvath and Gangl investigated the effect of spark energy on the mass concentration of carbon particles produced in a modified SDG (Horvath and Gangl 2003). They observed a tendency similar to that is shown in figure 7B exhibiting a threshold energy value after which the mass concentration of particles starts to saturate. They report a threshold energy of about $10 \mathrm{~mJ}$ for carbon particles (Horvath and Gangl 2003). This suggests that the electrode erosion tendencies revealed by OES are consistent with the literature and describe a general process that depends on the exact conditions of nanoparticle production and the material of the electrodes and hence the generated NPs.

\section{Conclusions}

In the present work, we performed optical emission spectroscopic measurements on the plasma of a spark discharge nanoparticle generator (SDG) and complemented those with particle characterization data for the generation of copper and gold nanoparticles (NPs) in nitrogen atmosphere.

It was shown for $\mathrm{Cu}$ that the time-resolved emission spectra can be used to derive the temporal variation of plasma temperature. From the rate of temperature drop in the cooling (afterglow) phase of the process, the quenching rate of the $\mathrm{Cu}$ atoms was derived to be $(5.5 \pm 0.6) \times 10^{8} \mathrm{Ks}^{-1}$. Such a fast quenching implies that the metal vapour generated in the first approx. $6 \mu \mathrm{s}$ of the process cools down to about room temperature in as short as $\sim 25 \mu$ s after the onset of the breakdown. This result provides a rough estimate for the temporal starting point of $\mathrm{Cu}$ NP generation, which is crucial for particle growth models.

Agglomerated $\mathrm{Cu}$ and $\mathrm{Au}$ NPs generated during the operation of the SDG were sampled by an SMPS. The variation of the mobility diameter and the peak concentration of the generated NPs were investigated 
as a function of two control parameters of the SDG, namely the gap size and the charging current of the capacitor. $\mathrm{Cu}$ and $\mathrm{Au}$ particles with modal diameter in the range of $18-30 \mathrm{~nm}$ and $21-45 \mathrm{~nm}$ were generated, respectively. Both the peak concentration and the modus of the size distribution of the generated particles increase linearly with increasing charging current via the variation of the spark repetition rate (SRR). The tendencies obtained for the variation of the electrode gap show a peak size at $2 \mathrm{~mm}$ and $3 \mathrm{~mm}$ with mobility diameters of $34 \mathrm{~nm}$ and $42 \mathrm{~nm}$ for $\mathrm{Cu}$ and $\mathrm{Au}$, respectively, which is the result of the combined effect of SRR and spark energy.

The emitted light of the spark was spectrally resolved and acquired with $50 \mathrm{~ns}$ temporal résolution. The initial stages of the temporal evolution of the spark plasma are characterized by the intense emission of the atoms and ions of the carrier gas. As the current flowing in the spark gap ceases, atomic spectral lines of the electrode material and the gas atmosphere start to dominate the emission spectrum. The erosion of the electrode material takes place in the arc stage of the discharge, more specifically in the first $6 \mu$ s and followed by the cooling of the metal vapour in the afterglow stage, which lasts for about $25 \mu$ s after the onset of the breakdown. It was shown how the optical emission of species, collected during the cooling of the metal vapour, varies when changing the key control parameters (i.e. charging current and gap size) of the SDG. Furthermore, it was also shown that the changes in the optical emission are determined by the energy per spark, regardless of whether it was achieved via changing the spark gap or the charging current. The intensity of spectral lines of atomic $\mathrm{N}$ increases linearly with increasing energy per spark. However, the intensity of emission lines of metal atoms - both $\mathrm{Cu}$ and $\mathrm{Au}$ - proceeds towards a plateau beyond a threshold energy. This suggests that the concentration of metal vapour produced in the SDG can only be increased up to a threshold value via increasing the spark energy, above which it levels off, ultimately setting a limit to the size and concentration of the NPs generated in the process.

The energy corresponding to the gap size at which the mobility diameter and the concentration of the generated NPs peak, coincides neatly with the threshold energy determined from the optical emission of metal atoms. This is our most direct evidence for the strong correlation between the optical emission of the spark plasma and the properties of the NPs generated in the SDG. These results demonstrate the suitability of OES as a valuable characterization tool that will allow for the more deliberate optimization of spark-based NP generation.

\section{Acknowledgements}

The research leading to these results has received funding from the European Union's Seventh Framework Program under Grant Agreement No. 280765 (BUONAPART-E) and was also supported by the GINOP-2.3.2-15-2016-00036 ("Development and application of multimodal optical nanoscopy methods in life and materials sciences") project.

\section{References}

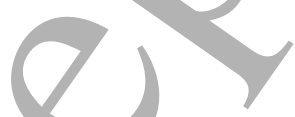

Aragón C and Aguilera J A 2008 Characterization of Laser Induced Plasmas by Optical Emission Spectroscopy: a Review of Experiments and Methods Spectrochim. Acta B 63 893-916

Bae Y, Pikhitsa P V, Cho H and Choi M 2017 Multifurcation Assembly of Charged Aerosols and Its Application to 3D Structured Gas Sensors Adv. Mater. 291604159

Berkowitz A E and Walter J L 1987 Spark Erosion: A Method for Producing Rapidly Quenched Fine Powders J. Mater. Res. 2 277-288 
Biskos G, Vons V, Yurteri C U and Schmidt-Ott A 2008 Generation and Sizing of Particles for AerosolBased Nanotechnology KONA Powder Part. J. 26 13-35

Boddu S R, Gutti V R, Ghosh T K, Tompson R V and Loyalka S K 2011 Gold, Silver, and Palladium Nanoparticle/Nano-Agglomerate Generation, Collection, and Characterization J. Nanopart. Res. 13 6591-6601

Borra J-P 2006 Nucleation and Aerosol Processing in Atmospheric Pressure Electrical Discharges: Powders Production, Coatings and Filtration J. Phys. D. 39 R19-R54

Borra J-P, Goldman A, Goldman M and Boulaud D 1998 Electrical Discharge Regimes and Aerosol Production in Point-To-Plane DC High Pressure Cold Plasmas: Aerosol Production by Electrical Discharges J. Aerosol Sci. 29 661-674

Boumans P W J M 1972 Excitation of Spectra. In Analytical Emission Spectroscopy ed. E L Grove (New York: Marcel Dekker)

BUONAPART-E: www.buonapart-e.eu

Byeon J H, Park J H and Hwang J (2008) Spark Generation of Monometallic and Bimetallic Aerosol Nanoparticles J. Aerosol Sci. 39 888-896

Byeon J H, Park J H, Yoon K Y and Hwang J 2009 Ambient Spark Generation to Synthesize CarbonEncapsulated Metal Nanoparticles in Continuous Aerosol Manner Nanoscale 1 339-343

Crawford F W and Edels H 1960 The Reignition Voltage Characteristics of Freely Recovering Arcs. Proc. IEE 107 202-212

Feng J, Biskos G and Schmidt-Ott A 2015 Toward Industrial Scale Synthesis of Ultrapure Singlet Nanoparticles with Controllable Sizes in a Continuous Gas-Phase Process Sci. Rep. 515788

Feng J, Huang L, Ludvigsson L, Messing M E, Maisser A, Biskos G and Schmidt-Ott A 2016 General Approach to the Evolution of Singlet Nanoparticles from a Rapidly Quenched Point Source $J$. Phys. Chem. C 120 621-630

Fridman A and Kennedy L A 2011 Plasma Physics and Engineering (2nd edition) (Boca Raton: CRC Press)

Galbács G 2006 A Review of Applications and Experimental Improvements Related to Diode Laser Atomic Spectroscopy Appl. Spectrosc. Rev. 41 259-303

Helsper C, Molter W, Löffler F, Wadenpohl C, Kaufmann S and Wenninger G 1993 Investigations of a New Aerosol Generator for the Production of Carbon Aggregate Particles Atmos. Environ. A 27 $1271-1275$

Hontanón E, Palomares J M, Stein M, Guo X, Engeln R, Nirschl H and Kruis F E 2013 The Transition from Spark to Arc Discharge and its Implications with respect to Nanoparticle Production $J$. Nanopart. Res. 151957

Horvath H and Gangl M 2003 A Low-Voltage Spark Generator for Production of Carbon Particles $J$. Aerosol Sci. 34 1581-1588

Jenkins N T and Eagar T W/ 2003 Submicron Particle Chemistry: Vapor Condensation Analogous to Liquid Solidification JOM 55 44-47

Kala S, Theissmann R, Rouenhoff M and Kruis F E 2016 Metal-Semiconductor Pair Nanoparticles by a Physical Route Based on Bipolar Mixing Nanotechnology 27125604

Kato M 1976 Preparation of Ultrafine Particles of Refractory Oxides by Gas Evaporation Method Jpn. J. Appl. Phys. 15 757-760 
Kohut A, Galbács G, Márton Zs and Geretovszky Zs 2017 Characterization of a Copper Spark Discharge Plasma in Argon Atmosphere used for Nanoparticle Generation Plasma Sources Sci. Technol. 26 045001

Ludvigsson L, Meuller B O and Messing M E 2015 Investigations of Initial Particle Stages During Spark Discharge J. Phys. D. 48314012

Magnusson M H, Deppert K, Malm J O, Bovin J O and Samuelson L 1999 Size Selected Gold Nanoparticles by Aerosol Technology Nanostruct. Mater. 12 45-48

Mandelstam S (1959) Excitation of the Spectrum in a Spark Discharge Spectrochim. Acta 15 255-271

Martinen H and Tholl H 1970 Untersuchung Der Temperatur Und Der Expansion von Funkenkanalen in H2 Bei Variabler Energiezufuhr Z. Naturforsch. 25 430-439

Messing M E, Hillerich K, Bolinsson J, Storm K, Johansson J, Dick A K and Deppert K (2010a) A Comparative Study of the Effect of Gold Seed Particle Preparation Method on Nanowire Growth Nano. Res. 3 506-519

Messing M E et al. 2010b Generation of Pd Model Catalyst Nanoparticles by Spark Discharge J. Phys. Chem. C 114 9257-9263

Meuller B O, Messing M E, Engberg, D L J, Jansson A M, Johansson L I M, Norlén S. M, Tureson N and Deppert K 2012 Review of Spark Discharge Generators for Production of Nanoparticle Aerosols Aerosol Sci. Technol. 46 1256-1270

Muntean A, Wagner M, Meyer J and Seipenbusch M 2016 Generation of Copper, Nickel, and CuNi Alloy Nanoparticles by Spark Discharge J. Nanopart. Res. 18229

Nilsson P T et al. 2015 In-Situ Characterization of Metal Nanoparticles and their Organic Coatings Using Laser Vaporization Aerosol Mass Spectrometry Nano Res. 8 3780-3795

NIST: http://physics.nist.gov/PhysRefData/ASD/lines form.html

Palomares J M, Kohut A, Galbács G, Engeln R and Geretovszky Zs 2016 A Time-Resolved Imaging and Electrical Study on a High Current Atmospheric Pressure Spark Discharge J. Appl. Phys. 118 233305

Pfeiffer T V, Feng J and Schmidt-Ott A 2014 New Developments in Spark Production of Nanoparticles Adv. Powder Technol. 25 56-70

Pfeiffer T V, Kedia P, Messing M E, Valvo M and Schmidt-Ott A 2015 Precursor-Less Coating of Nanoparticles in the Gas Phase Materials 8 1027-1042

Raizer Y P 1991 Gas Discharge Physics (Berlin Heidelberg: Springer-Verlag)

Scheeline A 1990 Sampling Processes in Emission Spectroanalytical Chemistry Mikrochim. Acta I 247285

Scheeline A and Coleman D M 1987 Direct Solids Elemental Analysis: Pulsed Plasma Sources Anal. Chem. 59 1185-1196

Schmidt-Ott A, Schurtenberger, P and Siegmann H C 1980 Enormous Yield of Photoelectrons from Small Particles Phys. Rev. Lett. 45 1284-1287

Schwyn S, Garwin E and Schmidt-Ott A 1988 Aerosol Generation by Spark Discharge J. Aerosol Sci. 19 639-642

Tabrizi N S, Ullmann M, Vons V A, Lafont U and Schmidt-Ott A 2009 Generation of Nanoparticles by Spark Discharge J. Nanopart. Res. 11 315-332

Wagner M, Kohut A, Geretovszky Zs, Seipenbusch M and Galbács G 2016 Observation of Fine-Ordered Patterns on Electrode Surfaces Subjected to Extensive Erosion in a Spark Discharge J. Aerosol Sci. $9316-20$ 


\section{From plasma to nanoparticles: optical and particle emission of a spark discharge generator

1

2

3

4

5

6

7

8

9

10

11

12

13

14

15

16

17

18

19

20

21

22

23

24

25

26

27

28

29

30

31

32

33

34

35

36

37

38

39

40

41

42

43

44

45

46

47

48

49

50

51

52

53

54

55

56

57

58

59

60

Walters J P 1969 Historical Advances in Spark Emission Spectroscopy Appl. Spectrosc. 23 317-331

Walters J P and Malmstadt H V 1965 Emission Characteristics and Sensitivity in a High-Voltage Spark Discharge Anal. Chem. 37 1484-1503

Wegner K and Pratsinis S E 2003 Scale-Up of Nanoparticle Synthesis in Diffusion Flame Reactors Chem. Eng. Science 58 4581-4589

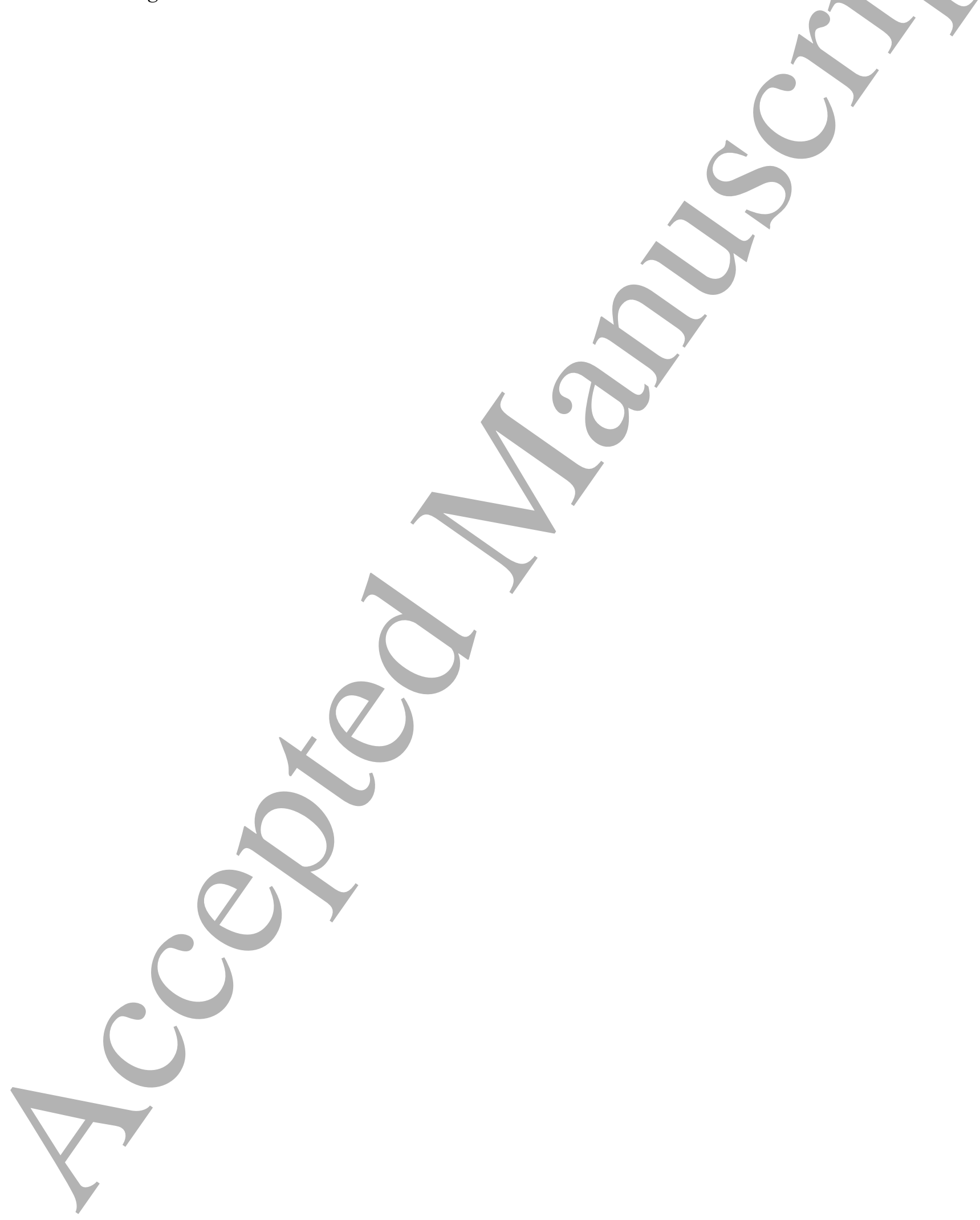

